\title{
Attitude of health care professionals about the diabetes from India
}

\author{
K. V. S. Hari Kumar, A. K. Gupta, Amit Kumar \\ Department of Endocrinology, Command Hospital, Lucknow, Uttar Pradesh, India
}

\section{A B S T R A C T}

Background: Health care workers should have adequate knowledge and the correct attitude to deal with the diabetes. We conducted this study among nursing and medical students to assess their attitude regarding diabetes. Materials and Methods: This cross-sectional descriptive study was conducted on medical (Group I, N = 50) and nursing (Group 2, N=50) students. Data was collected using the Diabetes Attitude Scale questionnaire developed by the University of Michigan Diabetes Research and Training Centre. The scores identify the attitude regarding five key areas of diabetes, namely need for special training, seriousness of diabetes, value of tight control, psychosocial impact and patient autonomy. The data were analyzed with appropriate statistical tests using GraphPad Prism Software, version 6 (GraphPad Software, San Deigo, CA, USA). Results: The study participants consist of 100 students of which 27 were male and 73 were female with a mean age of $22.9 \pm 3.3$ year. The scores in any subscale did not differ between the medical and nursing students. The correlation analyzes revealed that seriousness of diabetes is increasing linearly with age $(P=0.0325)$ and all the five key areas of diabetes showed a significant correlation with the increasing duration of residency $(P<0.05)$. Conclusion: Increasing age and more exposure to formal education are the major determinants of an individual's attitude toward diabetes. Our study attempts to highlights the need of education for all health care workers involved in the management of diabetes.

Key words: Attitude, diabetes mellitus, health care workers, student survey

\section{INTRODUCTION}

Diabetes mellitus (DM) is a spectrum of diseases characterized by hyperglycemia and associated metabolic abnormalities. The disease is divided into type 1 diabetes mellitus characterized by insulin deficiency and type 2 diabetes mellitus (T2DM) due to insulin resistance. T2DM is assuming pandemic proportions in the developing countries and is responsible for enormous morbidity and mortality. ${ }^{[1]}$ India leads among the countries with the highest number of diabetes patients with population

\begin{tabular}{|l|l|}
\hline \multicolumn{2}{|c|}{ Access this article online } \\
\hline Quick Response Code: & Website: \\
\hline & www.joshd.net \\
\hline & \\
\hline
\end{tabular}

prevalence of around $10-15 \% .{ }^{[2]}$ Management of diabetes and associated complications pose great challenges to the health care workers. The burden of the disease is huge on the health care sector in all countries. ${ }^{[3,4]}$ Developing countries like India have more problems with meager allocation of resources and the need to share the burden with communicable diseases also. ${ }^{[5]}$ Extraordinary efforts are required from all the health care givers to prevent the morbidity and mortality associated with diabetes.

Education and awareness about the diabetes is essential as it correlates with better outcomes leading to improved quality adjusted life years. ${ }^{[6-8]}$ Management of any chronic disease is a complex model and involves equal participation of health care practitioners, nurse educators, paramedical personnel, medical students and the patients. ${ }^{[9-11]}$ Medical and nursing students have limited exposure in understanding the methodology to deal with a chronic disease. They are the future caregivers and it is essential to assess their knowledge about diabetes which may reflect the management of disease in the future. Their knowledge,

Corresponding Author: Dr. K. V. S. Hari Kumar, Department of Endocrinology, Command Hospital, Lucknow - 226002 , Uttar Pradesh, India. E-mail: hariendo@rediffmail.com 
attitude and awareness about the disease play a vital role in the dissemination of information about diabetes. The studies evaluating the attitude of diabetes amongst medical students are limited from our country. ${ }^{[12]}$ Hence, we conducted this study amongst nursing and medical students to assess the attitude regarding diabetes.

\section{MAterials And Methods}

This cross-sectional descriptive study was conducted at a tertiary level hospital in India. The hospital runs a postgraduate program in 11 medical specialties affiliated to Medical Council of India toward an MD/MS degree. Our hospital also has an affiliated nursing school conducting a 3 year diploma courses. We carried out this survey among all postgraduate residents of various specialties $(N=50)$ and an equal number of nursing students. The study year (First, Second and Final) of each student is also recorded and is used for correlation analyzes. The participants were not allowed to consult each other while answering questions and the response sheets were collected after $30 \mathrm{~min}$. The participants were divided into two groups: Group 1 (medical) and Group 2 (nursing) students for the comparison.

The Diabetes Attitude Scale (DAS) questionnaire developed by the University of Michigan Diabetes Research and Training Center is given to all the participants and the responses were noted. The questionnaire consists of 33 questions and the participants are requested to respond on a 5 point scale that varies from complete agreement (score 5) to disagreement (score 1). ${ }^{[13]}$ The scores for the relevant questions were grouped to get the attitudes regarding five key areas of diabetes. They are (a) need for special training (b) seriousness of T2DM as a problem (c) value of tight control (d) psychosocial impact of DM and (e) patient autonomy. The local ethics committee approved the trial protocol and all the participants provided verbal informed consent.

Data are presented as mean \pm standard deviation and a comparison between the groups was carried out using nonparametric (Mann-Whitney U test) and Chi-square tests. Pearson's correlation test was used for correlation between numerical variables and a $P<0.05$ was considered to be significant. The statistical analysis and graph generation was carried out using the GraphPad Prism Software, Version 5 (GraphPad Software, San Deigo, CA, USA).

\section{RESULTS}

The study participants consist of 100 students (27 males and 73 females) with a mean age of $22.9 \pm 3.3$ year. The responses from the participants and the comparison between the groups are given in Table 1. Patients in Group 1 had higher age than when compared with nursing students. The responses were not different between both the groups for all parameters. The correlation analyzes revealed that seriousness of diabetes is increasing linearly with age as shown in Figure 1. All the five key areas of diabetes attitude showed a significant correlation with increasing duration of residency as shown in Table 2.

\section{DISCUSSION}

Our data showed that the medical and nursing students have a similar attitude toward diabetes. The five subscales of the DAS questionnaire showed similar results amongst the nursing and medical students. This is heartening to know that nursing students with limited exposure to diabetes have shown equal care and concern similar to that of medical students. Diabetes is seen in more than $1 / 3^{\text {rd }}$ of inpatients of any hospital and this could be leading to increased awareness of the diabetes amongst the nursing

\begin{tabular}{|c|c|c|c|c|}
\hline \multicolumn{5}{|c|}{$\begin{array}{l}\text { Table 1: Comparison between the groups regarding the five } \\
\text { key components of DAS }\end{array}$} \\
\hline Feature & Units & $\begin{array}{c}\text { Group } 1 \\
n=50\end{array}$ & $\begin{array}{c}\text { Group } 2 \\
n=50\end{array}$ & $P$ value \\
\hline Age & Years & $25.3(2.8)$ & $20.5(1.5)$ & $<0.0001$ \\
\hline $\begin{array}{l}\text { Need for } \\
\text { special training }\end{array}$ & Score & $21.9(2.6)$ & $22(1.98)$ & 0.8319 \\
\hline $\begin{array}{l}\text { Seriousness of } \\
\text { T2DM }\end{array}$ & Score & $26.6(2.4)$ & $25.7(3.8)$ & 0.1675 \\
\hline $\begin{array}{l}\text { Value of tight } \\
\text { control }\end{array}$ & Score & $23.3(3.6)$ & $24(2.8)$ & 0.2675 \\
\hline $\begin{array}{l}\text { Psychosocial } \\
\text { impact of DM }\end{array}$ & Score & $23.1(3.1)$ & $22.8(2.7)$ & 0.5853 \\
\hline Patient autonomy & Score & $29.3(4.7)$ & $29.8(3.5)$ & 0.5652 \\
\hline
\end{tabular}

Mean (SD). DAS: Diabetes attitude scale; T2DM: Type 2 diabetes mellitus; DM: Diabetes mellitus; SD: Standard deviation

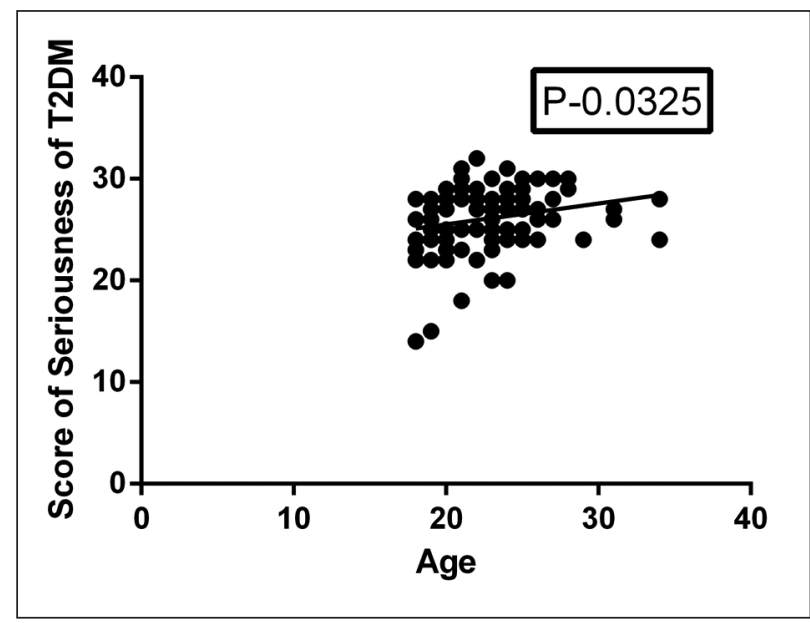

Figure 1: Correlation between seriousness of diabetes and age of the student 


\begin{tabular}{lcc}
\hline \multicolumn{3}{l}{ Table 2: Correlation analyses with age and year of residency } \\
\hline Individual question & Age & Study year \\
\hline Need for special training & $r=0.02621$ & $r=0.2612$ \\
& $P=0.7957$ & $P=0.0087$ \\
Seriousness of T2DM & $r=0.2140$ & $r=0.2131$ \\
& $P=0.0325$ & $P=0.0333$ \\
Value of tight control & $r=0.09185$ & $r=0.2467$ \\
& $P=0.3634$ & $P=0.0133$ \\
Psychosocial impact of DM & $r=0.1020$ & $r=0.2482$ \\
Patient autonomy & $P=0.3128$ & $P=0.0128$ \\
& $r=0.02751$ & $r=0.2113$ \\
& $P=0.7859$ & $P=0.0349$
\end{tabular}

T2DM: Type 2 diabetes mellitus; DM: Diabetes mellitus

students. ${ }^{[14]}$ The attitude of the patients is also important along with the caregivers to improve the outcomes. ${ }^{[15]}$ Direct public education and mass media campaigns are required to curb the rising trend of diabetes in India. ${ }^{[16]}$ The strategies should be implemented nationally to have an impact on the progression of the disease. This was demonstrated effectively in eradicating the infectious diseases like polio and similar strategy is required for noncommunicable diseases as well. ${ }^{[17]}$

The most important observation from our study is that all the five areas of the DAS questionnaire showed a correlation with the year of residency. The students in the final year of training felt the need for special training in diabetes and understood the concepts of tight control, patient autonomy etc. [Figure 2]. This could be because of the cumulative exposure to the diabetes in the curriculum and also at the place of work. The nursing diploma and postgraduate medical students have a 3 year residency program and diabetes touches most of the health care workers irrespective of the specialty. The prevention programs of diabetes emphasize the patient empowerment, education and community participation. ${ }^{[18-20]}$ Univariate correlation revealed that the seriousness of diabetes is increasing with age as seen in Figure 1. This could be a biased observation as the mean age is significantly different between both groups. Increasing age brings more experience and education which could also contribute to the heightened awareness about the diabetes. It is surprising that none of the remaining four variables showed any relation to the age of the student.

Education also played an important part in limiting the psychosocial impact of diabetes. Previous reports suggest that the stress and psychosocial impact of diabetes is more in lower socio-economic strata. ${ }^{[21]}$ The education of the patient is most important for the prevention of the morbidity and mortality associated with diabetes. ${ }^{[22,23]}$ Population studies have revealed that the awareness

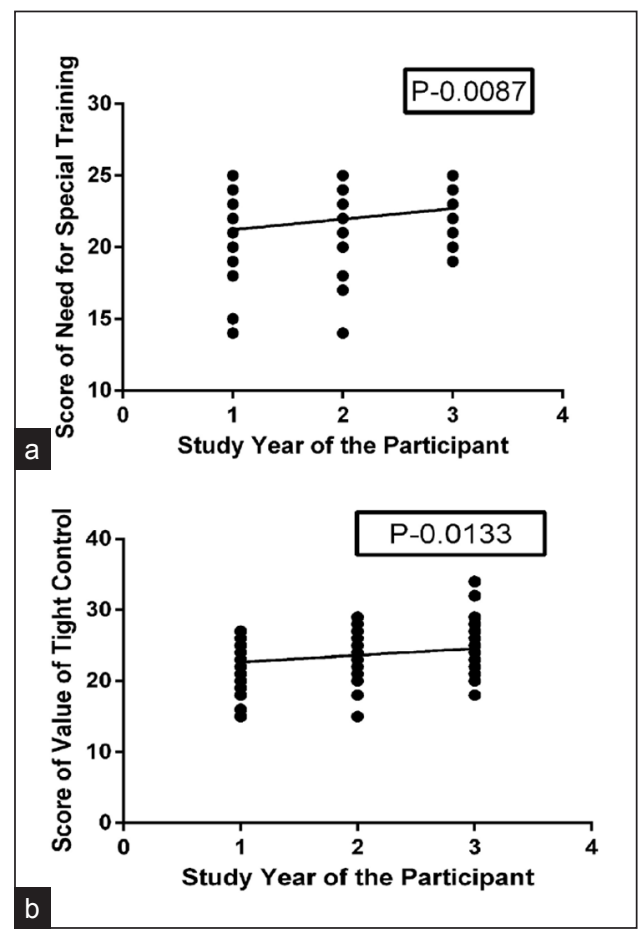

Figure 2: Correlation analyzes of training (a) and value of tight control (b)

about diabetes is very minimal in Indian population. ${ }^{[10]}$ This requires urgent attention from the policy makers with active participation of doctors and pharmaceutical industry. Education also helps in adopting a healthy lifestyle and eating patterns. ${ }^{[24]}$ The importance of life-style diseases should be included in the school curriculum to prevent a generation of obesity related diabetes in future adults. ${ }^{[25]}$ The limitations of our study are small sample size and all the study participants belong to the same institute. The data derived from a single center may not be a true representation of the population.

\section{CONCLUSION}

Nursing and medical students have shown similar trends regarding their knowledge and attitude about diabetes. The scores improved with increasing duration of residency and education. Further studies, involving more number of students are required to identify the attitudes of the future generation.

\section{REFERENCES}

1. Joshi SR, Saboo B, Vadivale M, Dani SI, Mithal A, Kaul U, et al. Prevalence of diagnosed and undiagnosed diabetes and hypertension in India - Results from the Screening India's Twin Epidemic (SITE) study. Diabetes Technol Ther 2012;14:8-15.

2. Somannavar S, Lanthorn H, Deepa M, Pradeepa R, Rema M, Mohan V. Increased awareness about diabetes and its complications in a whole city: Effectiveness of the "prevention, awareness, counselling and evaluation" [PACE] Diabetes Project 
[PACE-6]. J Assoc Physicians India 2008;56:497-502.

3. Lau SL, Debarm R, Thomas N, Asha HS, Vasan KS, Alex RG, et al. Healthcare planning in north-east India: A survey on diabetes awareness, risk factors and health attitudes in a rural community. J Assoc Physicians India 2009;57:305-9.

4. Shaini GS, Venkatesan L, Ben A. Effectiveness of structured teaching on home care management of diabetes mellitus. Nurs J India 2007;98:197-9.

5. Sayed SI, Dutta S, Mateen S, Kazi R, Jagade M. Clinical health research in India: Is there a way forward? J Indian Med Assoc 2011;109:270-1.

6. Mohan D, Raj D, Shanthirani CS, Datta M, Unwin NC, Kapur A, et al. Awareness and knowledge of diabetes in Chennai - The Chennai Urban Rural Epidemiology Study [CURES-9]. J Assoc Physicians India 2005;53:283-7.

7. Murugesan N, Snehalatha C, Shobhana R, Roglic G, Ramachandran A. Awareness about diabetes and its complications in the general and diabetic population in a city in southern India. Diabetes Res Clin Pract 2007;77:433-7.

8. Fatim J, Karoli R, Chandra A, Naqvi N. Attitudinal determinants of fasting in type 2 diabetes mellitus patients during Ramadan. J Assoc Physicians India 2011;59:630-4.

9. Kaur K, Singh MM, Kumar, Walia I. Knowledge and self-care practices of diabetics in a resettlement colony of Chandigarh. Indian J Med Sci 1998;52:341-7.

10. Singh A, Milton PE, Nanaiah A, Samuel P, Thomas N. Awareness and attitude toward diabetes in the rural population of Arunachal Pradesh, Northeast India. Indian J Endocrinol Metab 2012;16 Suppl 1:S83-6.

11. Pakenham-Walsh N, Bukachi F. Information needs of health care workers in developing countries: A literature review with a focus on Africa. Hum Resour Health 2009;7:30.

12. Das P, Basu M, Chowdhury K, Mallik S, Dhar G, Biswas A. Observational assessment and correlates to blood pressure of future physicians of Bengal. Niger J Clin Pract 2013;16:433-8.

13. Anderson RM, Fitzgerald JT, Funnell MM, Gruppen LD. The third version of the Diabetes Attitude Scale. Diabetes Care 1998;21:1403-7.

14. Noor Abdulhadi NM, Al-Shafaee MA, Wahlström R, Hjelm K. Doctors' and nurses' views on patient care for type 2 diabetes: An interview study in primary health care in Oman. Prim Health Care Res Dev 2013;14:258-69.

15. Rao PR, Shobhana R, Lavanya A, Padma C, Vijay V,
Ramachandran A. Development of a reliable and valid psychosocial measure of self-perception of health in type 2 diabetes. J Assoc Physicians India 2005;53:689-92.

16. Naeem AG. The role of culture and religion in the management of diabetes: A study of Kashmiri men in Leeds. J R Soc Promot Health 2003;123:110-6.

17. John TJ, Vashishtha VM. Eradicating poliomyelitis: India's journey from hyperendemic to polio-free status. Indian $\mathrm{J}$ Med Res 2013;137:881-94.

18. Balagopal P, Kamalamma N, Patel TG, Misra R. A communitybased participatory diabetes prevention and management intervention in rural India using community health workers. Diabetes Educ 2012;38:822-34.

19. Kalra S, Sridhar GR, Balhara YP, Sahay RK, Bantwal G, Baruah MP, et al. National recommendations: Psychosocial management of diabetes in India. Indian J Endocrinol Metab 2013;17:376-95.

20. Bajaj S, Jawad F, Islam N, Mahtab H, Bhattarai J, Shrestha D, et al. South Asian women with diabetes: Psychosocial challenges and management: Consensus statement. Indian J Endocrinol Metab 2013;17:548-62.

21. Mendenhall E, Shivashankar R, Tandon N, Ali MK, Narayan KM, Prabhakaran D. Stress and diabetes in socioeconomic context: A qualitative study of urban Indians. Soc Sci Med 2012;75:2522-9.

22. Chellan G, Srikumar S, Varma AK, Mangalanandan TS, Sundaram KR, Jayakumar RV, et al. Foot care practice - The key to prevent diabetic foot ulcers in India. Foot (Edinb) 2012;22:298-302.

23. Rani PK, Raman R, Agarwal S, Paul PG, Uthra S, Margabandhu G, et al. Diabetic retinopathy screening model for rural population: Awareness and screening methodology. Rural Remote Health 2005;5:350.

24. Kumar S, Mohanraj R, Sudha V, Wedick NM, Malik V, Hu FB, et al. Perceptions about varieties of brown rice: A qualitative study from Southern India. J Am Diet Assoc 2011;111:1517-22.

25. Shah P, Misra A, Gupta N, Hazra DK, Gupta R, Seth P, et al. Improvement in nutrition-related knowledge and behaviour of urban Asian Indian school children: Findings from the 'Medical education for children/Adolescents for Realistic prevention of obesity and diabetes and for healthy aGeing' (MARG) intervention study. Br J Nutr 2010;104:427-36.

How to cite this article: Kumar KH, Gupta AK, Kumar A. Attitude of health care professionals about the diabetes from India. J Soc Health Diabetes 2014;2:92-5.

Source of Support: Nil. Conflict of Interest: None declared. 